\title{
Variação do peso fresco em Cornops aquaticum (Bruner) (Orthoptera, Acrididae) associado a Eichhornia azurea (Sw) Kunth (Pontederiaceae) em uma baía no Pantanal de Poconé, Mato Grosso
}

\author{
Fatima R. Jaloretto da Silva ${ }^{1}$, Marinêz I. Marques ${ }^{1,2}$, Leandro D. Battirola ${ }^{3}$ \& Marcos G. Lhano ${ }^{4}$
}

\begin{abstract}
'Programa de Pós-Graduação em Ecologia e Conservação da Biodiversidade, Universidade Federal de Mato Grosso. Av. Fernando Corrêa da Costa, s/n, Coxipó, 78060-900 Cuiabá-MT, Brasil. fateca@gmail.com.br; marinez@ufmt.br

${ }^{2}$ Bolsista Produtividade em Pesquisa CNPq - Nível 2

${ }^{3}$ Instituto de Ciências Naturais, Humanas e Sociais, Universidade Federal de Mato Grosso, Campus Universitário de Sinop-MT, Brasil. ldbattirola@uol.com.br ${ }^{4}$ Centro de Ciências Agrárias, Ambientais e Biológicas - CCAAB, Universidade Federal do Recôncavo da Bahia, 44380-000 Cruz das Almas-BA, Brasil. marcos@ufrb.edu.br
\end{abstract}

\begin{abstract}
Variation of the fresh weight in Cornops aquaticum (Bruner) (Orthoptera, Acrididae) associated with Eichhornia azurea (Sw) Kunth (Pontederiaceae) in a bay in the Pantanal of Poconé, Mato Grosso. Cornops aquaticum (Bruner, 1906) (Orthoptera, Acrididae) develops its life cycle on aquatic macrophyte of the Pontederiaceae family. As grasshoppers are able to respond to the seasonal changes, the alternation of periods that occurs in the Pantanal may reflect in their biology. This study was carried out in the Pantanal of Poconé- MT, with the aim of evaluating possible variations in the fresh weight of the adults and nymphs of $C$. aquaticum. From March/2006 to February/2007, 50 individuals of $C$. aquaticum were collected each month. A total of 600 individuals was assessed, $43.5 \%$ of which were adults and $56.5 \%$ nymphs. The highest values of total fresh weight occurred in September (9.106g; 0.182g/individual) and October/2006 (8.865g; 0.177g/individual) and the lowest in March/2006 (3.413g; $0.068 \mathrm{~g} /$ individual). Among the adult individuals the highest fresh weight was registered in September $/ 2006(8.680 \mathrm{~g} ; 0.223 \mathrm{~g} /$ individual) and October/2006 (8.654g; 0.234g/individual), at the end of the dry period, and the lowest in March/2006 (1.792g; $0.138 \mathrm{~g} /$ individual) during the flood period. The nymphs had the highest fresh weight in April/2006 (2.913g; 0.076g/individual) at the beginning of the draining period, whereas the lowest fresh weight occurred in October $/ 2006(0.211 \mathrm{~g} ; 0.016 \mathrm{~g} /$ individual $)$ at the beginning of the flood period. Only the variation in the average fresh weight of females was significant $(\mathrm{f}=6.43 ; p=0.001)$ with the highest recordings occurring during the flood period, what may show a reproductive strategy.
\end{abstract}

KEYWORDS. Fresh weight; grasshoppers; population structure.

RESUMO. Variação do peso fresco em Cornops aquaticum (Bruner) (Orthoptera, Acrididae) associado a Eichhornia azurea (Sw) Kunth (Pontederiaceae) em uma baía no Pantanal de Poconé, Mato Grosso. Cornops aquaticum (Bruner, 1906) (Orthoptera, Acrididae) desenvolve seu ciclo de vida sobre macrófitas aquáticas da família Pontederiaceae. Como os gafanhotos são capazes de responder às mudanças sazonais, a alternância de períodos que ocorre no Pantanal pode refletir em sua biologia. Este estudo foi desenvolvido no Pantanal de Poconé - MT, com o objetivo de avaliar possíveis variações no peso fresco dos adultos e ninfas de $C$. aquaticum. Durante o período de março/2006 a fevereiro/2007 coletaram-se mensalmente, 50 indivíduos de C. aquaticum. Um total de 600 indivíduos foi avaliado, sendo 43,5\% adultos e 56,5\% ninfas. Os maiores valores de peso fresco total ocorreram nos meses de setembro (9,106g; 0,182g/indivíduo) e outubro/2006 (8,865g; 0,177g/indivíduo) e os menores em março/2006 (3,413g; 0,068g/ indivíduo). Nos indivíduos adultos os maiores pesos frescos foram registrados em setembro/2006 (8,680g; 0,223g/indivíduo) e outubro/2006 (8,654g; 0,234g/indivíduo), no final do período de seca, e o menor em março/2006 (1,792g; 0,138g/indivíduo), durante o período de cheia. As ninfas tiveram o maior peso fresco em abril/2006 (2,913g;0,076g/indivíduo) início da vazante, enquanto o menor peso fresco ocorreu em outubro/2006 (0,211g; $0,016 \mathrm{~g} /$ indivíduo $)$ início da enchente. Apenas a variação no peso fresco médio das fêmeas foi significativa ( $\mathrm{f}=6,43 ; p=0,001$ ), com os maiores registros durante o período de enchente, o que pode evidenciar uma estratégia reprodutiva.

PALAVRAS-CHAVE. Peso fresco; gafanhotos; estrutura populacional.

Cornops aquaticum (Bruner, 1906) (Orthoptera, Acrididae, Leptysminae) é uma espécie semi-aquática de gafanhoto distribuída desde o sudeste do México até a região central da Argentina e Uruguai (Adis et al. 2007; Capello et al. 2007). Esta espécie vive permanentemente associada à plantas aquáticas dos gêneros Eichhornia (Kunth) e Pontederia (L.), ambas Pontederiaceae (Carbonell 1981; Adis et al. 2007; Francheschini et al. 2007), e conforme Zolessi (1956) possui seu ciclo de vida estreitamente relacionado a Eichhornia crassipes (Mart.) Solms e E. azurea (Sw) Kunth.
Estudos evidenciam que outras macrófitas, aparentemente, não lhe proporcionam um substrato adequado para oviposição endofítica (Lhano et al. 2005).

As macrófitas aquáticas do gênero Eichhornia são originárias da América do Sul (Center et al. 2002), e foram introduzidas com finalidade ornamental em muitas áreas tropicais e subtropicais (Sato \& Testuya 1981), onde, por sua facilidade de dispersão e adaptabilidade, tornaram-se daninhas e de importância econômica, considerando os problemas causados tais como a obstrução de canais e reservatórios, 
abrigo para insetos vetores de doenças e diminuição do oxigênio disponível na água (Capello et al. 2007). Devido a estes problemas, diferentes tipos de controle, objetivando minimizá-los, foram testados incluindo herbicidas, controle mecânico e biológico (Oberholzer \& Hill 2001).

No Pantanal de Mato Grosso macrófitas aquáticas são bastante comuns devido à ocorrência de grandes áreas permanentemente inundadas, o que favorece a proliferação dessas planta e adaptação ao ambiente. Dentre as espécies mais freqüentes encontram-se as pontederiáceas, principalmente E. azurea. No Pantanal de Poconé, especificamente nas baías próximas à Rodovia Transpantaneira, esta espécie é uma das mais abundantes, formando extensos camalotes, conforme encontrado na baía Piuval (Fortney et al. 2004), local de realização deste estudo.

Considerando que $C$. aquaticum está estritamente associado a estas plantas e tem sido citado como inimigo natural das mesmas, já que suas ninfas e adultos causam grandes danos devido à herbivoria (Oberholzer \& Hill 2001), estudos tem apontado o seu papel como agente de controle biológico de macrófitas, sendo planejada a liberação desses gafanhotos em áreas ocupadas por estas plantas na África do Sul (Adis et al. 2007). Diante dessa possibilidade, estudos envolvendo esse gafanhoto tem sido desenvolvidos em diferentes partes do mundo para avaliar sua potencialidade (Capello et al. 2007; Franceschini et al. 2005; Lhano et al. 2005; Oberholzer \& Hill 2001).

O grande potencial dos gafanhotos como agentes de controle de plantas confronta-se ao fato destes insetos serem capazes de modificações adaptativas na alocação de recursos e exibirem respostas demográficas às mudanças na qualidade e disponibilidade das plantas hospedeiras (Branson 2006). Assim, são necessários estudos aprofundados que permitam avaliar possíveis variações que gerem impactos de sua utilização. Estes estudos devem abordar aspectos morfométricos e biométricos, bem como reconhecimento de possíveis fatores que possam influenciá-los em determinada região geográfica.

Desta maneira, esta pesquisa objetivou analisar as variações no peso fresco de ninfas e adultos de $C$. aquaticum ao longo de 12 meses, na baía Piuval no Pantanal de Poconé, Mato Grosso, visando contribuir para o conhecimento de aspectos biológicos e ecológicos desta espécie de gafanhoto na região, auxiliando a tomada de decisões com relação ao manejo e controle de macrófitas aquáticas em outras localidades.

\section{MATERIAL E MÉTODOS}

Área de estudo. As coletas foram realizadas na baía Piuval, localizada na Fazenda Ipiranga, no km 10 da Rodovia Transpantaneira, Pantanal de Poconé, Mato Grosso, no período compreendido entre março/2006 e fevereiro/2007. Esta baía caracteriza-se pela constante presença de água, mesmo nos períodos de secas extremas, e por apresentar grandes camalotes com predomínio de E. azurea. Durante o período de coleta a temperatura máxima registrada foi de $28,4^{\circ}$ $\mathrm{C}$ em novembro/2006 e a mínima de $19,5^{\circ} \mathrm{C}$ em julho/2006. A maior precipitação foi registrada em fevereiro/2007

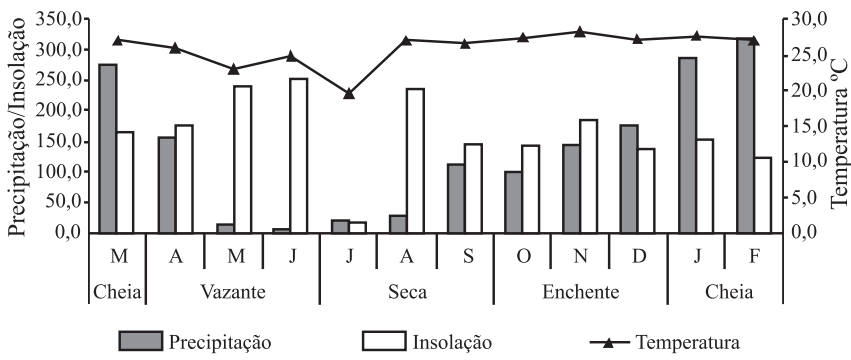

Fig. 1. Precipitação $(\mathrm{mm})$, insolação $(\mathrm{h} / \mathrm{s})$ e temperatura relativas ao período de março/2006 a fevereiro /2007 (Fonte: INMET-MT).

$(315,7 \mathrm{~mm})$, enquanto a menor ocorreu em junho/2006 (4,1 $\mathrm{mm})$. Com relação à insolação destaca-se que os maiores valores foram aferidos em junho/2006 (251,3 horas) e os menores em fevereiro/2007 (122,7 horas) (Fig. 1). Não foi possível a obtenção de dados exatos de insolação referente ao mês de julho/2006 devido a problemas técnicos no heliógrafo da estação meteorológica.

Metodologia. Para a realização deste estudo utilizouse o protocolo de coletas indicado pelo projeto "HICWA - Host Insect Coevolution Waterhyacinth" objetivando a futura comparação destes resultados com diferentes regiões geográficas. Este protocolo consiste na amostragem mensal de 50 gafanhotos (ninfas e adultos) ou até 30 minutos de coleta, caso não alcance logo os 50 exemplares, utilizando-se uma rede entomológica de $70 \mathrm{~cm}$ de diâmetro e fundo de $1 \mathrm{~m}$ de filó. A rede entomológica era posicionada sobre os bancos de E. azurea, com o barco em movimento, para a coleta dos insetos. No presente estudo este procedimento foi sempre inferior a 30 minutos, devido à abundância desta espécie, facilitando a captura do número de indivíduos necessários.

Os animais coletados foram colocados em sacos de plástico contendo folhas de E. azurea e transportados vivos, em caixa de isopor, até o Laboratório de Ecologia e Taxonomia de Artrópodes do Instituto de Biociências da Universidade Federal de Mato Grosso (UFMT), onde foram levados ao freezer até a morte. Após este procedimento todos os indivíduos foram separados em adultos (machos e fêmeas)

Bray-Curtis Cluster Analysis (Single Link)

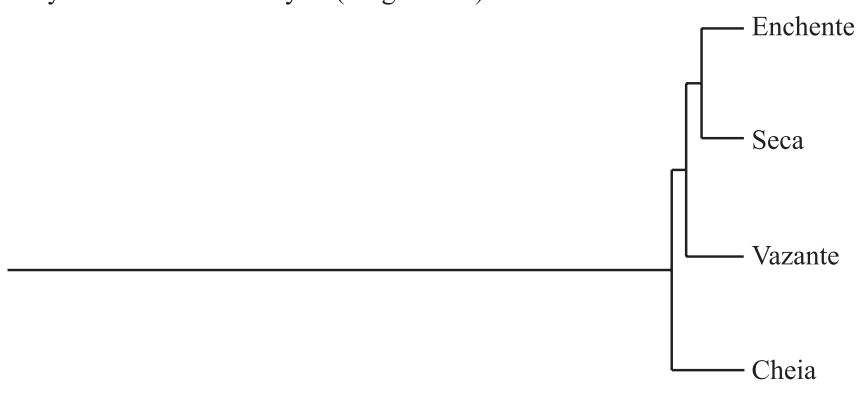

\begin{tabular}{ll|l}
\hline 0 & $\%$ Similarity & 100
\end{tabular}

Fig. 2. Comparação do peso fresco médio de adultos de C. aquaticum entre períodos (cheia, vazante, seca, enchente), calculado pelo Índice de BrayCurtis, na baía Piuval, Pantanal de Poconé - Mato Grosso. 
Bray-Curtis Cluster Analysis (Single Link)

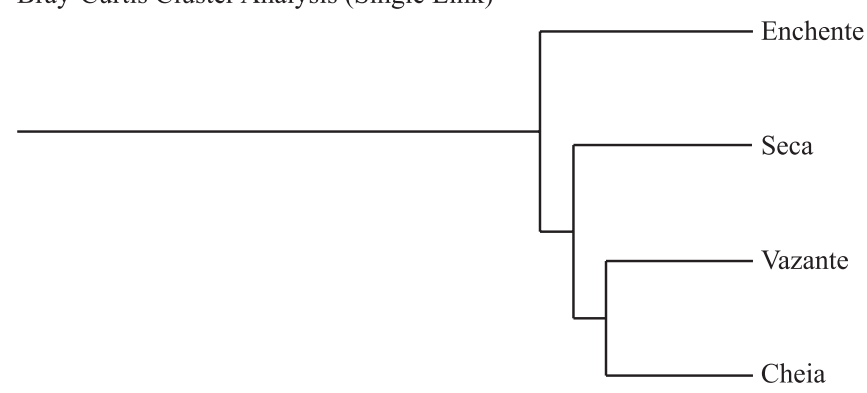

$0, \quad \%$ Similarity

50

100

Fig. 3. Comparação do peso fresco médio de ninfas de C. aquaticum entre períodos (cheia, vazante, seca, enchente), calculado pelo Índice de BrayCurtis, na baía Piuval, Pantanal de Poconé - Mato Grosso.

e ninfas. Dentre as ninfas a triagem foi efetuada em duas categorias: A - ninfa com até $12 \mathrm{~mm}$ de comprimento, que normalmente encontram-se nos estádios I, II e III e B - ninfas a partir de 12,5 mm de comprimento, estádios IV a VII. Todos os indivíduos, triados e descongelados, foram quantificados e pesados em balança analítica de precisão (Gehaka, $0,01 \mathrm{mg}$ ). Para verificação da variabilidade dos dados obtidos utilizouse como base a análise de variância $(\alpha=0,05)$, tendo como pressuposto de normalidade o teste de Levene. A similaridade entre os períodos sazonais foi avaliada pelo índice de BrayCurtis calculado pelo programa Biodiversity Profissional (McAleece et al. 1997).

\section{RESULTADOS E DISCUSSÃO}

Ao longo dos 12 meses de amostragem 600 indivíduos foram avaliados em relação ao peso fresco, dos quais 43,5 $\%$ representaram adultos (157 machos e 104 fêmeas) e 56,5 $\%$ ninfas (172 ninfas categoria A e 167 ninfas categoria B). Os maiores valores de peso fresco total (PFT) ocorreram nos

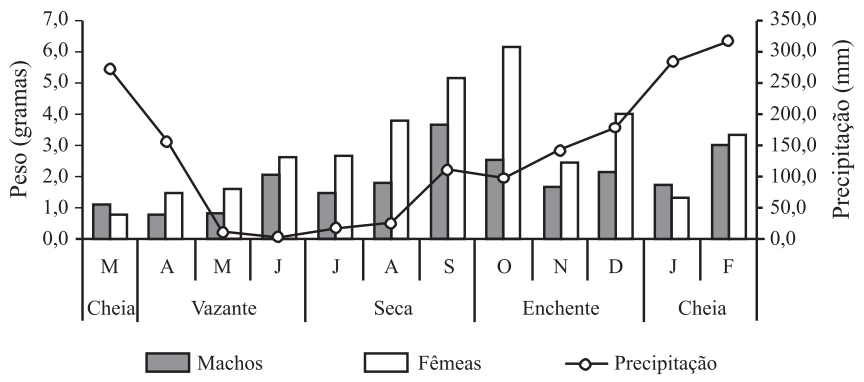

Fig. 4. Variação do peso fresco total (gramas) de adultos de C. aquaticum e a precipitação na baía Piuval, Pantanal de Poconé - Mato Grosso, durante o período de março/2006 a fevereiro/2007.

meses de setembro $(9,106 \mathrm{~g})$ e outubro de $2006(8,865 \mathrm{~g})$. Este resultado deve-se ao fato deste período apresentar a maior proporção de indivíduos adultos (setembro: 39 ind., 78,0 \%; outubro: 37 ind., 74,0 \%) na população, concomitante ao final do período de seca e início da enchente nesta região do Pantanal (Tabela I). Esta fase caracteriza-se como uma transição do período de escassez de chuvas para o período chuvoso, o que ocasiona alterações na estrutura do sistema pantaneiro e na umidade e temperatura, que podem influenciar diretamente o período reprodutivo destes insetos.

Com relação aos menores valores registrados, destacase o mês de março de 2006 com apenas 3,413g de peso fresco, que representa o final do período de cheia, quando se constatou a maior proporção de ninfas A e B (37 ind.; 74,0\%) em relação aos adultos (13 ind.; 26,0 \%) na população de C. aquaticum (Tabela I). Neste período também observou-se que a população de E. azurea era formada principalmente, por indivíduos jovens, que geralmente possuem folhas e pecíolos mais macios e atrativos aos insetos herbívoros em geral. Diferenças significativas não foram encontradas entre os períodos sazonais com relação à variação no peso fresco total $(\mathrm{f}=2,54 ; p=0,129)$, entretanto valores significativos ocorreram no padrão de peso fresco entre ninfas e adultos ( $\mathrm{f}=$ $19,8 ; p=0,001)$. A análise de similaridade entre os períodos sazonais também não demonstrou diferenças consideráveis,

Tabela I. Peso fresco (gramas) de C. aquaticum na baía Piuval, Pantanal de Poconé - Mato Grosso, no período de março/2006 a fevereiro/2007. (N = Número de indivíduos; PFT = Peso Fresco Total; PFM = Peso Fresco Médio; PMN = Peso Médio Ninfas; PMA = Peso Médio Adultos).

\begin{tabular}{|c|c|c|c|c|c|c|c|c|c|c|c|c|c|c|c|c|c|c|c|c|c|c|}
\hline \multirow{3}{*}{ Períodos } & & \multicolumn{9}{|c|}{ Ninfas } & \multicolumn{9}{|c|}{ Adultos } & \multirow{2}{*}{\multicolumn{3}{|c|}{ Total Geral }} \\
\hline & & \multicolumn{3}{|c|}{$\mathrm{A}$} & \multicolumn{3}{|c|}{ B } & \multicolumn{3}{|c|}{$\mathrm{A}+\mathrm{B}$} & \multicolumn{3}{|c|}{$\hat{0}$} & \multicolumn{3}{|c|}{ q } & \multicolumn{3}{|c|}{$\hat{0}+q$} & & & \\
\hline & & $\mathrm{N}$ & PFT & PFM & $\mathrm{N}$ & PFT & PFM & $\mathrm{N}$ & PFT & PMN & $\mathrm{N}$ & PFT & PFM & $\mathrm{N}$ & PFT & PFM & $\mathrm{N}$ & PFT & PMA & $\mathrm{N}$ & PFT & PFM \\
\hline Cheia & Mar. & 20 & 0,160 & 0,008 & 17 & 1,461 & 0,086 & 37 & 1,621 & 0,094 & 10 & 1,066 & 0,107 & 3 & 0,726 & 0,242 & 13 & 1,792 & 0,138 & 50 & 3,413 & 0,442 \\
\hline \multirow[t]{3}{*}{ Vazante } & Abr. & 12 & 0,230 & 0,019 & 26 & 2,683 & 0,103 & 38 & 2,913 & 0,122 & 7 & 0,722 & 0,103 & 5 & 1,393 & 0,279 & 12 & 2,115 & 0,176 & 50 & 5,028 & 0,504 \\
\hline & Maio & 19 & 0,182 & 0,010 & 19 & 2,043 & 0,108 & 38 & 2,225 & 0,117 & 7 & 0,761 & 0,109 & 5 & 1,567 & 0,313 & 12 & 2,328 & 0,194 & 50 & 4,553 & 0,539 \\
\hline & Jun. & 15 & 0,395 & 0,026 & 13 & 1,156 & 0,089 & 28 & 1,551 & 0,115 & 14 & 1,976 & 0,141 & 8 & 2,578 & 0,322 & 22 & 4,554 & 0,207 & 50 & 6,105 & 0,579 \\
\hline \multirow[t]{3}{*}{ Seca } & Jul. & 17 & 0,312 & 0,018 & 13 & 1,511 & 0,116 & 30 & 1,823 & 0,135 & 12 & 1,466 & 0,122 & 8 & 2,605 & 0,326 & 20 & 4,071 & 0,204 & 50 & 5.894 & 0,582 \\
\hline & Ago. & 24 & 0,328 & 0,014 & 4 & 0,138 & 0,035 & 28 & 0,466 & 0,048 & 11 & 1,804 & 0,164 & 11 & 3,717 & 0,338 & 22 & 5,521 & 0,251 & 50 & 5,987 & 0,550 \\
\hline & Set. & 5 & 0,053 & 0,011 & 6 & 0,373 & 0,062 & 11 & 0,426 & 0,073 & 24 & 3,586 & 0,149 & 15 & 5,094 & 0,340 & 39 & 8,680 & 0,223 & 50 & 9,106 & 0,562 \\
\hline \multirow[t]{3}{*}{ Enchente } & Out. & 9 & 0,068 & 0,008 & 4 & 0,143 & 0,036 & 13 & 0,211 & 0,043 & 19 & 2,516 & 0,132 & 18 & 6,138 & 0,341 & 37 & 8,654 & 0,234 & 50 & 8,865 & 0,517 \\
\hline & Nov. & 14 & 0,212 & 0,015 & 19 & 2,519 & 0,133 & 33 & 2,731 & 0,148 & 11 & 1,608 & 0,146 & 6 & 2,405 & 0,401 & 17 & 4,013 & 0,236 & 50 & 6,744 & 0,695 \\
\hline & Dez. & 7 & 0,425 & 0,061 & 20 & 1,571 & 0,079 & 27 & 1,996 & 0,139 & 13 & 2,101 & 0,162 & 10 & 3,955 & 0,396 & 23 & 6,056 & 0,263 & 50 & 8,052 & 0,696 \\
\hline \multirow[t]{2}{*}{ Cheia } & Jan. & 21 & 0,327 & 0,016 & 12 & 0,924 & 0,077 & 33 & 1,251 & 0,093 & 12 & 1,716 & 0,143 & 5 & 1,298 & 0,260 & 17 & 3,014 & 0,177 & 50 & 4,265 & 0,495 \\
\hline & Fev. & 9 & 0,196 & 0,022 & 14 & 0,880 & 0,063 & 23 & 1,076 & 0,085 & 17 & 2,943 & 0,173 & 10 & 3,260 & 0,326 & 27 & 6,203 & 0,230 & 50 & 7,279 & 0,584 \\
\hline Total & & 172 & 2,888 & 0,226 & 167 & 15,4 & 0,985 & 339 & 18,290 & 1,212 & 157 & 22,265 & 1,652 & 104 & 34,735 & 3,882 & 261 & 57,000 & 2,532 & $600^{\circ}$ & 75,290 & 6,745 \\
\hline
\end{tabular}




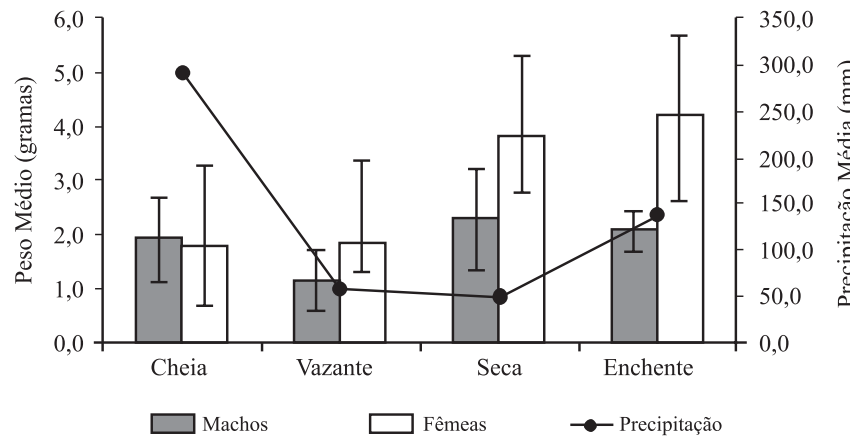

Fig. 5. Variação do peso fresco médio (gramas) de adultos de C. aquaticum e a precipitação média $(\mathrm{mm})$ na baía Piuval, Pantanal de Poconé - Mato Grosso, durante o período de março/2006 a fevereiro/2007.

com altos valores de similaridade entre estes períodos, tanto para adultos, quanto para ninfas (Figs. 2 e 3).

Avaliando-se separadamente os indivíduos adultos, observou-se que os maiores pesos frescos totais foram registrados em setembro/2006 $(8,680 \mathrm{~g})$ e outubro/2006 $(8,654 \mathrm{~g})$, meses caracterizados pelo final do período da seca e início da enchente, respectivamente. $\mathrm{O}$ menor peso fresco total ocorreu em março/2006 $(1,792 \mathrm{~g})$ durante o período de cheia (Tabela I; Fig.4). O peso fresco médio (PFM) calculado para machos foi de $0,142 \mathrm{~g}( \pm 0,02 \mathrm{~g})$, e de $0,334 \mathrm{~g}( \pm 0,05 \mathrm{~g})$ para as fêmeas, sendo que durante o período de coleta o menor peso médio encontrado para indivíduos machos foi de $0,103 \mathrm{~g}$ em abril/2006, e o maior de $0,173 \mathrm{~g}$ em fevereiro/2007, em fêmeas o menor peso médio foi em março/2006, 0,242g e o maior em novembro/2006, 0,401g (Tabela I). Neste estudo, a variação do peso fresco total entre os períodos não diferiu significativamente, assim como o peso fresco médio dos machos ( $\mathrm{f}=0,998 ; p=0,440$ ). Entretanto, ao analisar-se apenas a variação no peso fresco médio das fêmeas, observouse significância ( $\mathrm{f}=6,43 ; p=0,001)$, com os maiores registros durante o período de enchente (Fig. 5).

Esta diferença entre os períodos, evidenciada para as fêmeas, pode estar associada às adaptações fisiológicas dos insetos em relação às condições encontradas no ambiente, destacadas por Schowalter (2006). Como o Pantanal é uma região caracterizada pelas alterações sazonais propiciadas pelo regime hidrológico (Junk et al. 1989; 2006), é provável que a diferença de peso médio das fêmeas, de aproximadamente $65 \%(0,242 \mathrm{~g}$ na cheia e $0,401 \mathrm{~g}$ na enchente), represente uma estratégia reprodutiva para suportar um período de instabilidade como a cheia, quando a fitomassa de E. azurea é menor (Bini 1996). Estas adaptações podem estar associadas à maturação dos ovários de maneira sincronizada ao regime de secas e cheias do Pantanal, garantindo a sobrevivência da espécie. Estudos com gafanhotos terrestres demonstram que em certas populações ocorrem mudanças no peso das fêmeas, relacionadas com o período de oviposição, e que a falta de alimento pode levar à reabsorção de ovos pelos folículos, em um curto período de tempo em muitas espécies (Phipps 1966).

De acordo com a Tabela I, para as ninfas, obtevese $18,290 \mathrm{~g}$ de peso fresco total. As ninfas "A" tiveram o

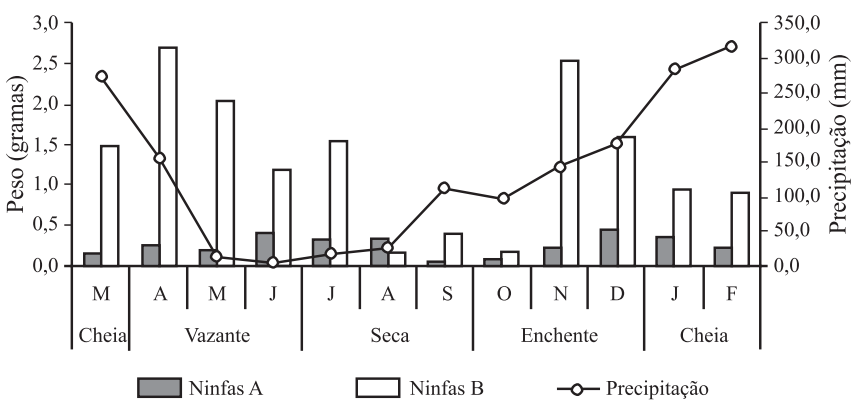

Fig. 6. Variação do peso fresco total (gramas) de ninfas A e B de C. aquaticum e a precipitação na baía Piuval, Pantanal de Poconé - Mato Grosso, durante o período de março/2006 a fevereiro/2007.

maior peso fresco em dezembro/2006 $(0,425 \mathrm{~g})$, final da enchente e início da cheia, enquanto o menor peso fresco foi registrado em setembro/2006 $(0,053 \mathrm{~g})$ durante o período de seca. Com relação aos pesos médios, obteve-se o maior valor em dezembro $(0,061 \mathrm{~g})$ devido à presença de ninfas de estádio de desenvolvimento mais adiantado (estádios II ou III), pois o número de indivíduos foi baixo $(\mathrm{N}=7)$. Para as ninfas "B", os maiores valores para peso fresco total foram aferidos em abril/2006 (2,683g), início da vazante, e novembro/2006 (2,519g), enchente (Tabela I, Fig. 6), provavelmente ocasionado pela presença de ninfas do estádio $\mathrm{V}$ ou VI. O menor valor para peso fresco total foi registrado em agosto/2006 (0,138g; 0,035g/indivíduo) durante a seca. A análise de variância não demonstrou significância na variação entre o peso fresco médio das ninfas em ambas as categorias $(\mathrm{A}-\mathrm{f}=0,488 ; p=0,699$ e $\mathrm{B}-\mathrm{f}=0,456 ; p=0,720)$.

Esta pequena variação no peso fresco médio das ninfas pode conjeturar as condições do ambiente, principalmente no que se refere à disponibilidade de alimento, pois, com o rebaixamento do nível da água durante os períodos de estiagem (vazante e seca), pode ocorrer alteração na disponibilidade de Eichhornia nestas áreas (Lhano et al. 2005). Bini (1996) relatou que os maiores valores de fitomassa de E. azurea ocorrem no período de menor nível d'água. Nessas condições os indivíduos adultos de $C$. aquaticum, devido à sua capacidade de vôo, encontram maior área de forrageamento, enquanto as ninfas, pela ausência de estruturas alares desenvolvidas, apresentam limitação para deslocarem-se em busca de alimento sendo, provavelmente, mais específicas com relação ao habitat.

Segundo Schowalter (2006), as adaptações morfológicas, fisiológicas e comportamentais dos insetos em geral, refletem as condições físicas do habitat que ocupam, bem como sua flutuação climática e distúrbios periódicos que afetam a sobrevivência dos organismos na comunidade. Como o Pantanal pertence à categoria das zonas úmidas temporárias, e, portanto, sujeitas a um previsível pulso de inundação monomodal (Junk et al. 2006), pode-se inferir que as espécies presentes neste ambiente demonstrem tal adaptação, como evidenciado nos valores de peso fresco obtidos nesse trabalho no período da seca e de enchente. 


\section{CONCLUSÃO}

A variação do peso fresco na população de C. aquaticum da baía Piuval não foi significativa nas análises efetuadas, porém, registraram-se diferenças entre o peso fresco médio de ninfas e adultos ao longo do período amostral, com amplitudes de peso de $0,043 \mathrm{~g}$ a $0,148 \mathrm{~g}$ em ninfas e $0,138 \mathrm{~g}$ a $0,263 \mathrm{~g}$ em adultos. Entretanto, foi verificada a distribuição desta população ao longo de todos os períodos sazonais, com alterações apenas em sua estrutura, com freqüências de ninfas e adultos em períodos alternados, acompanhando as oscilações do regime hidrológico. Os maiores pesos registrados para a população ocorreram durante o período de vazante, seca e começo da enchente, quando a precipitação é menor e provavelmente, a fitomassa de E. azurea é maior, evidenciando a forte associação com sua planta hospedeira.

Agradecimentos. Os autores dedicam este trabalho ao Prof. Dr. Joachim Adis (in memorian) pelo incentivo e grande dedicação aos estudos com Cornops aquaticum e pela constante atenção dispensada ao nosso grupo de estudos. Agradecemos também ao Sr. João Louzano (Fazenda Ipiranga), ao técnico Francisco Assis Gonçalves Rondon, aos colegas do Laboratório de Ecologia e Taxonomia de Artrópodes do Instituto de Biociências da Universidade Federal de Mato Grosso (UFMT), ao Instituto Nacional de Meteorologia - Cuiabá-MT pela concessão dos dados climáticos da região e ao Instituto Max-Planck para Limnologia, Plön, Alemanha pelo apoio logístico concedido para a realização deste estudo.

\section{REFERÊNCIAS}

Adis J.; E. Bustorf; M. G. Lhano; C. Amédégnato \& A. L. Nunes. 2007. Distribution of Cornops grasshoppers (Leptysminae: Acrididae: Orthoptera) in Latin America and the Caribbean Islands. Studies on Neotropical Fauna and Environment 42: 11-24.

Bini, L. M. 1996. Influência do pulso de inundação nos valores de fitomassa de três espécies de macrófitas aquáticas na planície de inundação do alto rio Paraná. Arquivo Biológico e Tecnológico 39: 715-721.

Branson, D. H. 2006. Life-history responses of Ageneotettix deorum (Scudder) (Orthoptera: Acrididae) to host plant availability and population density. Journal of the Kansas Entomological Society 79: 146-155.

Capello, S.; J. Adis \& M. L. de Wysiecki. 2007. Temperatura y fotoperíodo: qué influencia ejercen en el desarrollo ninfal de Cornops aquaticum (Orthoptera: Acrididae)? Amazoniana XIX: 209-216.
Carbonell, C. S. 1981. Arthropoda: Orthoptera, p. 92-99. In: S. H. Hulbertt, G. Rodrigues \& N. D. Santos, (eds.). Aquatic Biota of Tropical South America. San Diego, San Diego State University, 323 p.

Center, T. D; M. P. Hill; H. A. Cordo \& M. H. Julien 2002. Waterhyacinth, p. 41-64. In: R. Van Driesche; B. Blossey; M. Hoddle; S. Lyon \& R. Reardon. (eds.) Biological Control of Invasive Plants in the Eastern United States. West Virginia, USDA Forest Service Publication FHTET2002-04, $413 \mathrm{p}$.

Fortney, R. H.; M. Benedict; J. F. Gottgens; T. L. Walters; B. S. Leady \& J. Rentch. 2004. Aquatic plant community and distribution along an inundation gradient at two ecologically-distinct sites in the Pantanal region of Brazil. Wetlands Ecology and Management 12: 575-585.

Franceschini, M. C.; S. Capello; M. G. Lhano; J. Adis \& M. L. de Wysiecki. 2005. Morfometria de los estádios ninfales de Cornops aquaticum Bruner (1906) (Acrididae: Leptysminae) en Argentina. Amazoniana XVIII: $373-386$.

Franceschini, M. C.; J. Adis; A. P. de Neiff \& M. L. de Wysiecki. 2007. Fenologia de Cornops aquaticum (Orthoptera: Acrididae) en un camalotal de Eichhornia azurea (Pontederiaceae) en Argentina. Amazoniana XIX: 149-158.

Junk, W. J.; P. B. Bayley \& R. E. Sparks. 1989. The flood pulse concept in river-floodplain systems. Canadian Special Publications for Fisheries and Aquatic Sciences 106: 110-127.

Junk, W. J.; M. Brown; I. C. Campbell; M. Finlayson; B. Gopal; L. Ramberg \& B. G. Warner. 2006. The comparative biodiversity of seven globally important wetlands: a synthesis. Aquatic Sciences 68: 400-414.

Lhano, M. G.; J. Adis; M. I. Marques \& L. D. Battirola. 2005. Cornops aquaticum (Orthoptera, Acrididae, Leptysminae): aceitação de plantas alimentares por ninfas vivendo em Eichhornia azurea (Pontederiaceae) no Pantanal Norte, Brasil. Amazoniana XVIII: 397-404.

McAleece N.; P. J. D. Lambshead, G. L. J. Paterson \& J. D. Gage. 1997. Biodiversity Pro: Free Statistics Software for Ecology. The Natural History Museum \& The Scottish Association for Marine Science.

Oberholzer, I. G. \& M. P. Hill. 2001 How safe is the grasshopper Cornops aquaticum for release on water hyacinth in South Africa, p. 82-88. In: M. H. Julien; M. P. Hill; T. D. Center \& D. Jianqing.(eds.) Biological and integrated control of water hyacinth, Eichhornia crassipes. ACIAR Proceedings, $102 \mathrm{p}$.

Phipps, J. 1966. Ovulation and oocyte resorption in Acridoidea (Orthoptera). Proceedings of the Royal Entomological Society of London series A, 41: 78-86.

Sato, H. \& K. Testuya. 1981. Biomass production of waterhyacinth, and its ability to remove inorganic minerals from water. Japanese Journal of Ecology 31: 257-267.

Schowalter, T. D. 2006. Responses to abiotic conditions, p. 17-52. In: T. D. Schowalter (ed.) Insect Ecology. An Ecosystem Approach. 2 ed. Louisiana State University Baton Rouge: Academic Press, 572 p.

Zolessi, L. C. de. 1956. Observaciones sobre Cornops aquaticum BR (Acridoidea, Cyrtacanthacr.) en el Uruguay. Revista de la Sociedad Uruguaya de Entomologia 1: 3-28. 